\title{
Low levels of HDL-cholesterol and endothelial dysfunction: a systematic review and meta-analysis
}

Leonardo Roever, MHS ${ }^{1}$, Elmiro Santos Resende, $\mathrm{PhD}^{1}$, Angélica Lemos Debs Diniz, $\mathrm{PhD}^{1}$, Nilson Penha-Silva, PhD ${ }^{1}$, João Lucas $\mathrm{O}^{\prime}$ Connell, $\mathrm{PhD}^{1}$, Fernanda Rodrigues de Souza, $\mathrm{PhD}^{1}$, Poliana Rodrigues Alves Duarte, $\mathrm{PhD}^{1}$, Paulo Fernando Silva Gomes, $\mathrm{MD}^{1}$, Américo José Caixeta Neto ${ }^{1}$, Lívia Maria Ambrósio da Silva ${ }^{1}$, Rosangela de Oliveira Felice ${ }^{1}$, Hugo Ribeiro Zanetti, MSc ${ }^{1-2}$, Anaisa Silva Roerver-Borges, $\mathrm{MD}^{2}$, Fernando César Veloso, $\mathrm{MHS}^{1}$, Thiago Montes Fidale, $\mathrm{PhD}^{1}$, Antonio Casella-Filho, PhD ${ }^{3}$, Paulo Magno Martins Dourado, $\mathrm{PhD}^{3}$, Antonio Carlos Palandri Chagas, $\mathrm{MD}, \mathrm{PhD}^{3-4}$, Sadeq Ali-Hasan-Al-Saegh, MD ${ }^{5}$, Paulo Eduardo Ocke Reis, $\mathrm{PhD}^{6}$, Rogério de MeloCosta Pinto, $\mathrm{PhD}^{1}$, Gustavo B. F. Oliveira, $\mathrm{PhD}^{7}$, Álvaro Avezum, $\mathrm{PhD}^{7}$, Mansueto Neto, $\mathrm{PhD}^{8}$, André Durães, $\mathrm{PhD}^{8}$, Rose Mary Ferreira Lisboa da Silva, $\mathrm{PhD}^{9}$, Antonio José Grande, $\mathrm{PhD}^{10}$, CeliseDenardi, $\mathrm{MD}^{11}$, Renato Delascio Lopes $\mathrm{MD}$, $\mathrm{PhD}^{12}$, NiteshNerlekar, $\mathrm{MD}^{13}$, Shahab Alizadeh, $\mathrm{MSc}^{14}$, Adrian V. Hernandez, MD, $\mathrm{PhD}^{15}$, Maria Inês da Rosa, MD, $\mathrm{PhD}^{16}$, Gary Tse, $\mathrm{MD}$, PhD ${ }^{17}$, Tong Liu, MD, $\mathrm{PhD}^{18}$, Giuseppe Biondi-Zoccai, MD, MStat ${ }^{19}$

${ }^{1}$ Federal University of Uberlândia, Department of Clinical Research

${ }^{2}$ Heart Institute (InCor), Master Institute of Education President Antonio Carlos, IMEPAC, Araguari, Brazil, Department of Clinical Research ${ }^{3}$ HCFMUSP- University of São Paulo Medical School, Department of Cardiology, São Paulo, Brazil

${ }^{4}$ Faculty of Medicine ABC, Department of Cardiology Santo André, Brazil

${ }^{5}$ Cardiovascular Research Center, ShahidSadoughi University of Medical Sciences, Department of Cardiology, Yazd, Iran

${ }^{6}$ Department of Specialized and General Surgery, Fluminense Federal University, Rio de Janeiro, Brazil

${ }^{7}$ Dante Pazzanese Institute of Cardiology, Department of Clinical Research, São Paulo, Brazil

${ }^{8}$ Graduate Program in Medicine and Health, Department of Health and Sciences, Federal University of Bahia, Brazil

${ }^{9}$ Federal University of Minas Gerais, Department of Cardiology, MG, Brazil

${ }^{10}$ Federal University of Mato Grosso, Department of Medicine, MT, Brazil

${ }^{11}$ FOP Unicamp, Department of Clinical Research, SP. Brazil

${ }^{12}$ Division of Cardiology, Duke University Medical Center, Department of Clinical Research, Durham, NC, USA

${ }^{13}$ Monash Cardiovascular Research Centre and MonashHeart, Department of Cardiology, Clayton, Victoria, Australia

${ }^{14}$ Tehran University of Medical Sciences, Department of Medicine

${ }^{15}$ University of Connecticut/Hartford Hospital Evidence-Based Practice Center, Hartford, Department of Comparative Effectiveness and Outcomes Research Health Outcomes, CT, USA

${ }^{16}$ Laboratory of Epidemiology, University of Extremo Sul Catarinense, Criciúma, Brazil

${ }^{17}$ Department of Medicine and Therapeutics and Li Ka Shing Institute of Health Sciences, Faculty of Medicine, Chinese University of Hong Kong, Hong Kong, China

${ }^{18}$ Tianjin Key Laboratory of Ionic-Molecular Function of Cardiovascular Disease, Department of Cardiology, Tianjin Institute of Cardiology, the Second Hos-pital of Tianjin Medical University, Tianjin, China

${ }^{19}$ Department of Medico-Surgical Sciences and Biotechnologies, Sapienza University of Rome, Latina, Italy Department of AngioCardioNeurology, IRCCS Neuromed, Pozzilli, Italy

*Correspondence author: Dr. Leonardo Roever, MHS, Department of Clinical Research, Federal University of Uberlândia, Brazil; E-mail: leonardoroever@hotmail.com

Received: August 22, 2018; Accepted: September 04, 2018; Published: September 16, 2018

\section{Abstract}

\section{Introduction}

HDL-C is believed to retard the formation of atherosclerotic lesions by removing excess cholesterol from cells and preventing endothelial dysfunction. However, there are no systematic analyses or well-conducted meta-analyses to evaluate the relationship between very low HDL-C and endothelial dysfunction [1]. The aim of this study is to examine this association of very low HDL-C with endothelial dysfunction in different ages and sex.

\section{Methods and analysis}

The update systematic review and meta-analysis will be conducted using published studies that will be identified from electronic databases (i.e., PubMed, EMBASE, Web of Science, and Google Scholar. Studies that (1) examined the association between very low HDL-C and endothelial dysfunction, (2) had a longitudinal or prospective cohort design, (3) were conducted among in adults aged 34 to 70 years., (4) provided sufficient data for calculating ORs or relative risk with a 95\% CI, (5) were published as original articles written in English or other languages, and (6) have been published until January 2018 will be included. Study selection, data collection, quality assessment and statistical syntheses will be conducted based on discussions among investigators. 


\section{Ethics and dissemination}

Ethics approval was not required for this study because it was based on published studies. The results and findings of this study will be submitted and published in a scientific peer-reviewed journal.

\section{Strengths and limitations of this study}

This systematic review and meta-analysis will offer better understanding regarding the association between metabolic syndrome and endothelial dysfunction. The findings from this study will be useful for assessing of very low HDL-C and the risk factors in endothelial dysfunction, and determining approaches for prevention of endothelial dysfunction in the future.

An improved understanding of this relationship may help to inform public health endothelial dysfunction prevention strategies.

Included studies may have substantially different methodologies, which could limit our ability to draw reliable conclusions from the existing evidence base. Depending on the results, confounding factors that were not adjusted for the selected studies and low generalizability situations can be limitations.

To minimize these limitations we will evaluate the heterogeneity between the studies, perform sensitivity analysis and meta-regression.

Trial registration number: PROSPERO (CRD42018083467).

Abbreviations: $\mathrm{CIs}=$ Confidence Intervals, $\mathrm{HDL}=$ high-density lipoprotein, $\mathrm{LDL}=$ low-density lipoprotein, $\mathrm{MD}=$ mean difference, $\mathrm{RR}=$ risk ratio, $\mathrm{WC}=$ waist circumference

Key words: HDL-C, endothelial dysfunction, systematic review

\section{Background}

HDL is believed to retard the formation of atherosclerotic lesions by removing excess cholesterol from cells and preventing endothelial dysfunctionand a very low HDL-C increased risk of cardiovascular events [1].

It is in large part the results of unbalanced diet, low socioeconomic and cultural levels, stress and sedentary lifestyle. Although the literature on the very low HDL-C and the risk factors for endothelial dysfunction has been increasing, to our knowledge, a systematic review of the association between very low of HDL-C and risk of endothelial dysfunction has not yet been conducted [1-11].

This study aims to systematically access the association between a very low HDL-C and the endothelial dysfunction in adults aged 34 to 70 years; and to provide a framework to further understand these factors in order to better target prevention strategies.

\section{Methods/Design}

This systematic review of the literature will follow the Preferred Reporting Items for Systematic Reviews and Meta-Analyses (PRISMA) recommendations. The databases PubMed, Embase, Web of Science, Google Scholar, and Cochrane will be searched for articles [12]. Our search will focus on cohort, case-control and cross-sectional studies examining the association between very low HDL-C and endothelial dysfunction. The primary outcome is endothelial dysfunction. Two reviewers will independently screen articles, extract relevant data and assess the quality of the studies.

The aim of this analysis is to investigate whether there is an association between HDL-C levels and endothelial dysfunction in the adult population with cardiovascular outcomes. We plan to look at the prevalence of very low HDL-C levels in endothelial dysfunction individuals and to analyze whether low and very low HDL levels $(<20$; $20-30 ; 30-40$ vs. $>40 \mathrm{mg} / \mathrm{dL}$ as ref. according to sex) in endothelial dysfunction might to be additional risk factor and predictor of CVD events, and mortality (CV-mortality, mortality and all-cause mortality) [1-2].

The study is registered with PROSPERO (CRD42018083467). This protocol conforms to the Preferred Reporting Items for Systematic Reviews and Meta-Analyses Protocols (PRISMA-P) guidelines [13-14].

\section{Systematic review registration}

This protocol is registered in the PROSPERO registry of the University of York (Reference number: CRD42018083467).

\section{Objectives}

The primary objective is to identify and summarize the association with of very low of HDL-C levels and with endothelial dysfunction risk in adults (34-70 years) in different ages and sexes.

\section{Eligibility criteria}

The PICOS strategy (population, intervention (changed to exposure for the purposes of this review of observational studies), comparator, outcome, study characteristics) was used to define the eligibility criteria for this study.

Inclusion criteria: studies will be considered if they include: endothelial dysfunction patients in the diagnosis of the low and very low HDL levels $(<20 ; 20-30 ; 30-40$ vs. $>40 \mathrm{mg} / \mathrm{dL}$ as ref. according to sex).

Exclusion: Reviews or abstracts from congresses/conferences, letters, editorials, case reports, interventional studies or clinical trials. We excluded studies that did not provide information on low and very low HDL levels ( $<20 ; 20-30 ; 30-40$ vs. $>40$ as ref. according to sex) in endothelial dysfunction and a control group.

Data will be extracted using a standardized template. We will use the PICOS (Population, Intervention, Comparator, Outcomes and 
Study design) framework, originally devised to formulate a research question, as a basis to develop data extraction criteria. As this is an aetiological study, 'exposure' will replace 'intervention' and 'study characteristics' will replace 'study design'. Data items on the following five domains will be extracted:

1. Population: characteristics of the study population (e.g., mean/ median age, ethnic distribution), inclusion and exclusion criteria

2. Exposure: definition and identification of very low HDL-C.

3. Comparators: definition and identification of unexposed individuals, number of unexposed subjects

4. Outcomes: definition and identification of primary (HDLconcentration of $+/$ - endothelial dysfunction is the main exposure of interest. Patients with a diagnosis of endothelial dysfunction that present CVD outcomes, and death (CV mortality, all-cause mortality) and secondary outcomes (Biomarkers of endothelial cell dysfunction and endothelial cell activation, as well as noninvasive techniques to measure endothelial function), number of subjects with outcome

5. Study characteristics: authors, publication year, setting/source of participants, design, methods of recruitment and sampling, period of study, length of follow-up time (if relevant), aims and objectives.

\section{Outcomes}

\section{Primary outcomes}

HDL-concentration of $+/$ - endothelial dysfunction is the main exposure of interest. Patients with a diagnosis of endothelial dysfunction that present CVD outcomes, and death (CV mortality, all-cause mortality)

\section{Secondary outcomes}

Biomarkers of endothelial cell dysfunction, endothelial cell activation, as well as non-invasive techniques to measure endothelial function.

\section{Study design}

This is a systematic review and meta-analysis protocol of prospective cohort studies, following the PRISMA-P (Preferred Reporting Items for Systematic Reviews and Meta-Analysis protocols) guideline [14]. The systematic review and meta-analysis will be reported according to the PRISMA (Preferred Reporting Items for Systematic Reviews and Meta-Analyses) guideline [15]. The whole process of study selection is summarized in the PRISMA flow diagram (Fig. 1). This study will not involve any private patient data; ethics approval was waived (see online supplementary file 1 for PRISMA-P checklist).

\section{Search strategy}

A systematic review of the literature will be conducted. A language restriction shall not be applied to the search. If there are relevant non-English abstracts, attempts shall be made to translate them wherever possible. The following bibliographic databases (Embase, PubMed-MEDLINE, Web of Science, Cochrane Library, and Google
Scholar) will be searched for articles published until January 2018. The search strategy will be developed by LR and HRZ; we anticipate that the databases will be searched from their inception to 30 December 2018 (see online supplementary file1 for the search strategies for PubMed, EMBASE, Cochrane Library, Web of Science, Embase, Google Scholar.

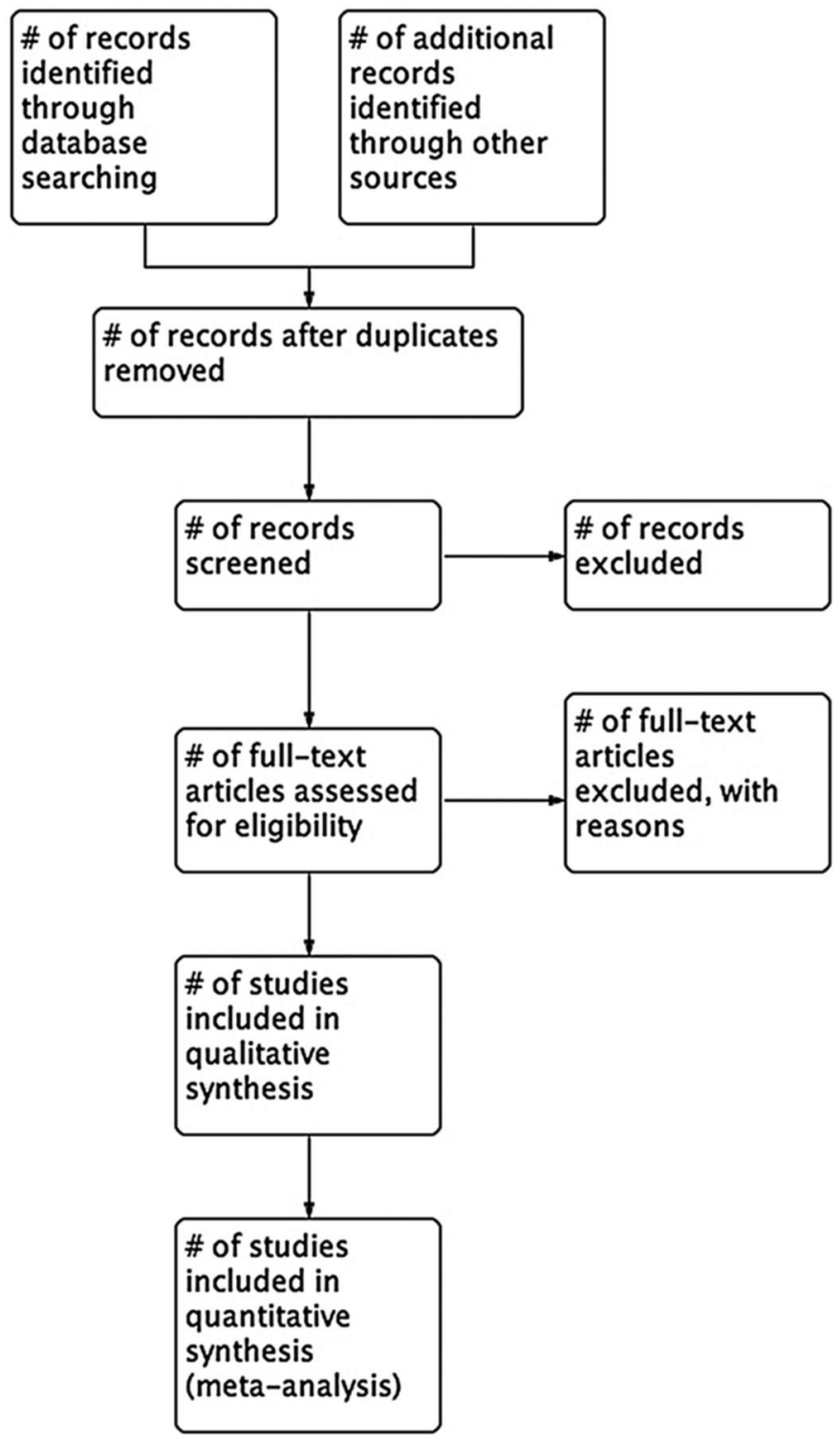

Figure 1. Flow diagram of study selection process

Our search focuses on studies examining the association between very low HDL-C diagnosis and endothelial dysfunction in adults (34-70 years) [11]. At each step of the selection process, reasons for inclusion/exclusion will be recorded in the PRISMA Flowchart [13].

\section{Data collection}

A record will be kept of all searches and search decisions to ensure reproducibility. Search results will be exported to a citation management program (EndNote ver. 7.0). Duplicates will be removed and retained separately. The resulting references will be exported separately to the two reviewers for independent review using MS Excel. 


\section{Selection of studies}

Two authors (LR, FCV) will independently screen all titles and abstracts identified through the literature searches and will exclude all records clearly not meeting inclusion criteria. Disagreements will be resolved by consensus. The selection process will be pilot tested to ensure a high degree of agreement between reviewers. Full text of the remaining studies will then be retrieved. The same two authors (LR, FCV) will independently assess the papers for fulfilment of inclusion criteria. In case of differences of opinion regarding study inclusion, a third review author (GBZ) will serve as arbiter. To avoid double counting, if multiple publications based on the same cohort of participants are retrieved, only the study reporting the largest sample size will be used. The reasons for excluding papers for which the full text was retrieved will be documented.

\section{Data extraction and management}

A data extraction form will be used to collect details from the included studies. The form includes information on study design, patient population, and presence of stroke. Two review authors (LR and FCV) will independently extract the data. The data extraction form will be pilot tested on several papers to ensure consistency and that all relevant information is being captured. If necessary, a statistician will review the extraction of data to further ensure quality and reliability. Authors will be contacted for missing data.

In terms of the study results, unadjusted and fully adjusted effect estimates for the association between very low HDL-C and endothelial dysfunction will be recorded. Details of the confounders measured and adjusted for will also be noted. Results of any additional stratified analyses will also be recorded. Where possible, results from additional subgroup analyses with evidence regarding our nonprimary objectives will also be recorded, for example, the association between very low HDL-C and the secondary outcomes.

\section{Assessment of methodological quality}

Two investigators (LR and FCV) will independently assess each selected study for study quality using the Newcastle-Ottawa Quality Assessment Scale (NOS) [16]. The NOS evaluates cohort studies based on eight items categorized into the following three groups: (1) selection of the study cases, (2) comparability of the population, and (3) ascertainment of whether the exposure or outcome includes any risk of bias (i.e., selection bias or bias from lost to follow-up). The NOS is scored ranging from 0 to 9 , and studies with scores $\geq 7$ are considered as high quality [16]. Discrepancy of quality assessment among the investigators will be solved by discussion and consensus among all authors.

\section{Data synthesis and statistical analysis}

We anticipate that there may be significant heterogeneity in the prevalence of very low HDL-C features of endothelial dysfunction. There are several factors that could contribute to such heterogeneity. The relative risk (RR), and odds ratio (OR) are the way the result will be expressed statistically.

These factors include the following: differences in demographic and clinical features (e.g., age, hypertension, renal disease, smoking, duration and severity of diabetes) among study cohorts; differences in definitions of HDL-C. An $\mathrm{I}^{2}$ statistic will be calculated for the studies to be included in each proposed meta-analysis (i.e. for each neuroradiology correlate of interest) with values of 25,50 , and $75 \%$ suggesting low, moderate, or high degrees of heterogeneity, respectively, which report a dichotomized (i.e., present or absent) or categorical (i.e., absent, mild, moderate, severe) shall be harmonized for meta-analysis if deemed appropriate by our statistician. Other types of rating scales shall not be included in a meta-analysis and the data based on any such data scale would be presented in narrative form.

If significant heterogeneity between studies, as determined by consultation with our statistician, prevents meaningful pooling of the data, we will limit ourselves to providing a narrative description of observed trends. Given the heterogeneity of the populations studied, assumption of a fixed effect size across populations would not be justified, thus analyses would be performed using a random effects model. Given the dichotomized (presence or absence) or categorical (severity measure) nature of our data of, meta-analysis will be performed a random effects analysis. We will also add funnel graphs, publication bias analysis and a meta-regression analysis.

If there are sufficient data to allow such analyses (in principle from as few as a single high quality study, but if possible by pooling data from multiple studies), we will perform subgroup analyses for participants with renal disease and participants with hypertension. In addition, if sufficient data are available, we shall perform subgroup analyses by age and diabetes duration. Funding sources and conflict of interest will be extracted from included studies. Statistical analysis will be performed using RevMan software.

\section{Strategy for data synthesis}

The data of interest presented as continuous (mean value and $\mathrm{SD}$ ) will be used to perform meta-analysis to obtain the standardized mean difference (SMD) and 95\% confidence interval (CI). Cocharn's Q-statistic and I-squared test will be used to test for heterogeneity between the included studies. If a I-squared value will be greater than $50 \%$ or a $\mathrm{p}$ value of the Q-test will be less than 0.05 , indicating maximal heterogeneity among the included studies, a random-effect model will be put into use.

\section{Analysis of subgroups or subsets}

The subgroup meta-analyses will be conduct according to the prespecified study-level characteristics using a fixed-effects meta-analysis and if there is substantial heterogeneity, we will use the random effects model. The sources included location, sex, age, method of HDL-C assessment, the definition of endothelial dysfunction. We also will conduct sensitivity analyses to evaluate the potential sources of heterogeneity in the analyses.

\section{Summary of Evidence}

We will produce a narrative synthesis of the main results extracted from articles in full text. A summary of the included studies will provide information on the authors, study design, participants, number and age of the subjects, theoretical structure (if relevant), alcohol consumption 
(as primary outcome of interest), main findings, Study information. Special emphasis will be placed on the identification of very low of HDL-C and the risk of endothelial dysfunction. In the presentation of the results, we will try to separate the factors for which the evidence of causality is strong (from longitudinal studies) and factors for which the causal nature of the relationship is less secure (cross-sectional data). A graphical summary of all the data they represent will be provided and take into account the number of studies that provide evidence of a factor and the relative strength of the association presented based on study design and quality assessment. The membership level will be evaluated based on adjusted data.

\section{Discussion}

This systematic review will synthesize research evidence to establish whether the risk of developing endothelial dysfunction is relatively high in adults with very low HDL-C. Strengths and limitations will be highlighted in the identified evidence. Strength of observational data may include large sample size, high rate of follow-up and frequency of stroke more likely to be representative of the population at risk. Limitations may include the quality of data extracted which may not allow studies to be combined in a meta-analysis. This may be overcome by presenting the findings in a descriptive manner. This review will conducted in collaboration with an experienced librarian who helped appraise the search criteria, refine the keywords and MeSH terms and identify appropriate database(s). To the best of our knowledge, no reviews have been published exploring the study question; however, if a review addressing a similar question is published, it will be incorporated in this review and added in a meta-analysis if feasible.

\section{Implications of results}

This systematic review will provide an updated and quantifiable estimate of the risk of endothelial dysfunction in adults with very low HDL-C. Furthermore, the systematic search will identify where future research is required. For instance, this review may inform a prognostic study which may be useful in understanding the course and factors associated with endothelial dysfunction development.

\section{Amendments}

If it is necessary we will update this protocol in the future. We will submit the original protocol, final protocol and summary of changes as a supplement.

\section{Ethics and dissemination}

\section{Ethical issues}

No ethical approval is required because this study includes no confidential personal data or interventions with the patients.

\section{Publication plan}

The procedures of this systematic review and NAM will be conducted in accordance with the PRISMA-compliant guideline. The results of this systematic review and NAM will be submitted to a peerreviewed journal for publication.

Authors' information: Not applicable
Competing interests: The authors declare that they have no competing interests.

Consent for publication: Not applicable

Ethics approval and consent to participate: Not applicable

Availability of supporting data: Not applicable

Funding: Not applicable

\section{Authors' contributions}

LR, ASRB, ALDD, ACF, NPS, PMMD, RMLS, JLO, MN, AD, PARD, FRS, GBFO, GBZ, SAH, PEOR, AJG, RMP, ACF, PMMD, TMF, NN, SA, CD, TL GT, AJCN, LMAS, ROF, PFSG, A.A, AVH, MIR, RDL and FCV conceived the study idea and devised the study methodology. LR, ASRB, ACPC and ESR participated in the design and coordination of the study. LR was primarily responsible for protocol writing and developed the search strategy. LR and FCV will screen identified literature, conduct data extraction and analyses the review findings. All authors read the drafts, provided comments and agreed on the final version of the manuscript.

Acknowledgements: Not applicable

\section{References}

1. Ahmed ST, Rehman H, Akeroyd JM, et al. (2018) Premature Coronary Heart Disease in South Asians: Burden and Determinants. Curr Atheroscler Rep 20: 6. [crossref]

2. Oliveira GBF, Avezum A, Roever L. Cardiovascular disease burden: evolving knowledge of risk factors in myocardial infarction and stroke through populationbased research and perspectives in global prevention. Front Cardiovasc Med. 2015: 200032.

3. Roever L, Biondi-Zoccai G, Chagas AC (2016) Non-HDL-C vs. LDL-C in Predicting the Severity of Coronary Atherosclerosis. Heart Lung Circ 25: 953-954. [crossref]

4. Roever LS, Resende ES, Diniz AL, Penha-Silva N, Veloso FC, et al. (2016) Abdominal Obesity and Association With Atherosclerosis Risk Factors: The Uberlândia Heart Study. Medicine (Baltimore) 95: e1357. [crossref]

5. Roever L, Resende ES, Diniz ALD, et al. Ectopic adiposopathy and association with cardiovascular disease risk factors: The Uberlândia Heart Study. Int J Cardiol. 2015; 190: 140-142.

6. Roever L, Resende ES, Veloso FC, Diniz AL, Penha-Silva N, et al. (2015) Perirenal Fat and Association With Metabolic Risk Factors: The Uberlândia Heart Study. Medicine (Baltimore) 94: e1105. [crossref]

7. Van Rooy MJ, Pretorius E. Metabolic syndrome, platelet activation and the development of transient ischemic attack or thromboembolic stroke.Thromb Res. 2015;135(3): 434-42.

8. Sarrafzadegan N, Gharipour M, Sadeghi M, Nezafati P, Talaie M, et al. (2017) Metabolic Syndrome and the Risk of Ischemic Stroke. J Stroke Cerebrovasc Dis 26: 286-294.

9. Sánchez-Iñigo L, Navarro-González D, Fernández-Montero A, Pastrana-Delgado J, Martínez JA. Risk of incident ischemic stroke according to the metabolic health and obesity states in the Vascular-Metabolic CUN cohort.Int J Stroke. 2017;12(2): 187-191.

10. Chei CL, Yamagishi K, Tanigawa T, Kitamura A, Imano H, Kiyama M, et. al. Metabolic Syndrome and the Risk of Ischemic Heart Disease and Stroke among Middle-Aged Japanese.Hypertens Res. 2008 Oct;31(10): 1887-9.

11. Fang X, Liu H, Zhang X, Zhang H, Qin X, Ji X. Metabolic Syndrome, Its Components, and Diabetes on 5-Year Risk of RecurrentStroke among Mildto-Moderate Ischemic Stroke Survivors: A Multiclinic Registry Study.J Stroke Cerebrovasc Dis. 2016; 25(3): 626-34

12. Glasziou P, Irwig L, Bain C, Colditz G. Systematic reviews in health care: a practical guide. Cambridge: Cambridge University Press; 2001.

13. Newcastle Ottawa Scale (The Newcastle-Ottawa Scale (NOS) for assessing the quality of nonrandomised studies in meta-analyses), available at: http: //www.ohri. ca/programs/clinical_epidemiology/oxford.asp. Accessed 26 Jan 2017.

14. Moher D, Liberati A, Tetzlaff J, Altman DG.Preferred reporting items for systematic reviews and meta-analyses: the PRISMA statement.BMJ. 2009;339. 
15. Shamseer L, Moher D, Clarke M, Ghersi D, Liberati A, Petticrew M, Shekelle P, Stewart L, PRISMA-P Group. Preferred reporting items for systematic review and meta-analysis protocols (PRISMA-P) 2015: elaboration and explanation.BMJ. 2015;349(jan02 1): g7647.
16. Wells GA, Shea B , O'Connell D, et al . The Newcastle-Ottawa Scale (NOS) for assessing the quality of nonrandomized studies in meta-analyses. Secondary The Newcastle-Ottawa Scale (NOS) for assessing the quality of nonrandomized studies in met analyses. http: //www.ohri.ca/programs/clinical epidemiology/oxford.asp (accessed on Dec 28, 2017)

\section{Citation:}

Leonardo Roever, Elmiro Santos Resende, Angélica Lemos Debs Diniz, Nilson Penha-Silva, et al. (2018) Low levels of HDL-cholesterol and endothelial dysfunction: a systematic review and meta-analysis. Internal Med Res Open J Volume 3(3): 1-6. 